\title{
Maternal mental health and child nutritional status in an urban slum community in Bangladesh
}

\section{Ahad Mahmud Khan ( $\nabla$ ahad_mahmud@hotmail.com )}

The University of the Edinburgh, Edinburgh, UK https://orcid.org/0000-0002-4347-0825

\section{Research Article}

Keywords: maternal mental health, common mental disorders, CMD, SRQ-20, child nutrition

Posted Date: November 29th, 2021

DOI: https://doi.org/10.21203/rs.3.rs-65789/v2

License: (c) (i) This work is licensed under a Creative Commons Attribution 4.0 International License. Read Full License 


\title{
Title
}

Maternal mental health and child nutritional status in an urban slum community in Bangladesh

\begin{abstract}
Objective: Poor mental health may diminish a mother's capacity to adequately care for her child, resulting in a negative impact on the child's nutrition. This study aims to determine the association between maternal mental health and child nutritional status in a poor urban population in Bangladesh.
\end{abstract}

Methods: We carried out a cross-sectional study among 264 mother-child pairs in an urban slum area of Bangladesh. The Self-Reporting Questionnaire-20 (SRQ-20) was used to assess maternal mental health. An SRQ-20 score $\geq 7$ was considered a common mental disorder (CMD). Anthropometric measurements were performed to assess child nutritional status.

Results: The prevalence of maternal CMD was 46.2\%. Maternal CMD was associated with poorer child feeding practice $(\mathrm{p}<0.001)$, poorer hygiene practice $(\mathrm{p}<0.001)$, poorer preventive care service use $(\mathrm{p}=0.016)$ and suffering from diarrheal disease $(\mathrm{p}=0.049)$. The prevalence of stunting, wasting and underweight was $44.3 \%$, $18.2 \%$ and $33.7 \%$, respectively. Poorer child feeding practice was associated with wasting $(\mathrm{p}=0.004)$ and underweight $(\mathrm{p}<0.001)$ but not with stunting. Poorer hygiene practice and suffering from diarrheal disease were associated with stunting and underweight but not with wasting. In multivariate analysis, maternal CMD was associated with child wasting $(\mathrm{AOR}=2.25,95 \% \mathrm{CI}=1.15-4.43)$. Association between maternal $\mathrm{CMD}$ and child underweight found in bivariate analysis was attenuated and no longer statistically significant after multivariate analysis $(\mathrm{AOR}=1.77,95 \% \mathrm{CI}=0.94-3.33)$. No statistically significant association was observed between maternal $\mathrm{CMD}$ and stunting in this study $(\mathrm{AOR}=1.46,95 \% \mathrm{CI}=0.84-2.54)$.

Conclusions: Maternal mental health affects child nutritional status through child feeding practice, hygiene practice and preventive care use. Interventions to address the mental health of mothers in child nutrition programs might contribute to improving child nutritional status.

\section{Keywords}

maternal mental health, common mental disorders, CMD, SRQ-20, child nutrition 


\section{Introduction}

Malnutrition is a leading cause of child mortality, morbidity and lifelong developmental impairment (Smith \& Haddad, 2000). Globally, 162 million under-five children are stunted; 99 million are underweight and one million are wasted; a major portion of these children reside in Asia (World Health Organization, 2012). Although the trends in malnutrition prevalence continue to decrease in recent years in Bangladesh, it is still very high (UNICEF, 2009). According to the Bangladesh Demographic and Health Survey (BDHS) 2011, the prevalence of stunting, wasting and underweight was 41\%, 16\% and 36\%, respectively (NIPORT, Mitra and Associates, \& ICF International, 2012). The contributing factors of child malnutrition include child age, sex, birth weight, previous birth interval, parents' education, maternal nutrition, low socioeconomic status, child feeding practice, hygiene, child illness, health seeking behavior, etc. (Black et al., 2013; Pongou, Ezzati, \& Salomon, 2006; Rayhan \& Khan, 2006).

Maternal mental health plays a major contributing role in child nutritional status (Ashaba, Rukundo, Beinempaka, Ntaro, \& LeBlanc, 2015; Cavalcante-Neto, Paula, Florencio, \& Miranda, 2016; Harpham, Huttly, De Silva, \& Abramsky, 2005; Nguyen et al., 2014; Santos, Santos, Silva Rde, Hasselmann, \& Barreto, 2011). Common mental disorders (CMDs) such as anxiety, depression, and somatic symptoms (Prince et al., 2007) are more frequent among women than men (Steel et al., 2014). CMDs are fairly common among mothers of underfive children, especially in developing countries (Harpham et al., 2005; Nguyen et al., 2014). In a multi-country study, the prevalence of maternal CMDs ranged from $21 \%$ in Vietnam to $33 \%$ in Peru (Harpham et al., 2005). In Bangladesh, the prevalence has been reported to be $49 \%$ (Nguyen et al., 2014).

When the mother's mental health is compromised, it adversely affects child nutrition (Ashaba et al., 2015; Cavalcante-Neto et al., 2016; Harpham et al., 2005; Nguyen et al., 2014; Santos et al., 2011), interfering with a mother's ability to care for her child (Surkan, Kennedy, Hurley, \& Black, 2011). Maternal CMD affects mothers' caregiving practices leading to improper feeding and inadequate health care for children especially immunization (Harpham et al., 2005; R. C. Stewart, 2007). Because of maternal CMD, hygiene practice is not properly maintained resulting in child illness especially diarrhea (Nguyen et al., 2014). There is a bidirectional relationship between infection and malnutrition. Infection causes malnutrition by reducing dietary intake and intestinal absorption, whereas malnutrition increases the risk of diarrhea as it can predispose to infection due to a weak immune system (Brown, 2003). Insufficient childcare doubles the probability of child malnutrition (Carvalhaes \& Benicio, 2006).

Several studies have been published on the association between maternal CMD and child nutritional status (Ashaba et al., 2015; Harpham et al., 2005; Medhin et al., 2010; Nguyen et al., 2014; Rahman, Iqbal, Bunn, Lovel, \& Harrington, 2004; Santos et al., 2011). However, there is a scarcity of literature focusing on the poor urban community where the prevalence of maternal CMD is elevated (Barsisa, Derajew, Haile, Mesafint, \& Shumet, 2021). Therefore, this study was designed to assess the relationship between maternal mental health and child nutritional status in the urban slum areas of Bangladesh. This result would help understand the complexities of the relationship between maternal mental health and child nutrition and strengthen the knowledge base of policy makers in decision making to combat child malnutrition. 


\section{Methods}

\section{Study design and setting}

The present study was a community-based cross-sectional study carried out from September to November 2013. It was conducted in an urban slum area at Kamrangichar of Dhaka district in Bangladesh. An estimated 400,000 population lived in its surface area of $3.68 \mathrm{~km}^{2}$. The study area is divided into 12 administrative areas or 'mohallas'.

\section{Study population and sample size}

The study population included mothers and their under-five children. Currently pregnant mothers were excluded because pregnancy has a potential effect on mother's mental health (Parcells, 2010) as well as her body mass index (BMI) (Olson, Strawderman, \& Dennison, 2009). Data collection was done from a sample of 264 motherchild pairs. The sample size was estimated considering the prevalence of child stunting (41\%) in Bangladesh (NIPORT et al., 2012) and allowing the study $80 \%$ power at a 5\% significance level in detecting associations with an odds ratio (OR) of 2 for maternal CMD. An equal number of participants were recruited from each 'mohalla' of the study area to achieve the sample size. Starting from the center of the 'mohalla', the data collectors approached a randomly selected direction. They knocked on doors of consecutive households, and if both mother and child were present at that period, they were recruited. In case of multiple under-five children of a mother, the youngest one was enrolled.

\section{Measurements}

A structured questionnaire was used that included questions on sociodemographic information, childcare practices and child illness. There were also questions from Self-Reporting Questionnaire-20 (SRQ-20) (World Health Organization, 1994), Household Food Insecurity Access Scale (HFIAS) (Coates, Swindale, \& Bilinsky, 2007), and Kuppuswamy’s socioeconomic status scale (Kumar, Gupta, \& Kishore, 2012). A checklist for height, weight and hygiene was used. According to study objectives, the variables were identified, and English questionnaire was drafted. After translating into local language (Bangla) and adapting for local context, the questionnaire was pretested on six respondents of similar background. After necessary modifications, the questionnaire was finalized.

\section{Self-Reporting Questionnaire-20}

The SRQ-20 developed by the World Health Organization (WHO) was used to measure maternal mental health. It is a 20-item tool that includes questions about depressive, anxiety, panic and somatic symptoms in the preceding four weeks. Each of the 20 items is scored 1 or 0 which indicates the presence or absence of symptoms, respectively. The sum of scores generates an overall SRQ-20 scale ranging from 0 to 20, where higher scores indicate poor mental health states and vice versa (World Health Organization, 1994). A cut-off of 7 was set to categorize women with 'CMD' or 'no CMD', as suggested by several studies (Harpham et al., 2005; Medhin et al., 2010; Nguyen et al., 2014; Robert C Stewart et al., 2008). The reliability and validity of this instrument are well established (World Health Organization, 1994) and it has been used in several studies 
including Bangladesh (Harpham et al., 2005; Medhin et al., 2010; Nguyen et al., 2014; Robert C Stewart et al., 2008).

\section{Household Food Insecurity Access Scale}

The HFIAS 9-item questionnaire was used to measure household food security which is appropriate for the urban population. The questionnaire asks a specific condition related to the experience of food insecurity for a period of four weeks preceding the survey. According to their HFIAS questionnaire scores, households were categorized into four groups: food secure, mildly, moderately and severely food insecure (Coates et al., 2007). It has been used in different surveys, including Bangladesh, and the reliability and validity are well established (Coates et al., 2007; Nguyen et al., 2014).

\section{Kuppuswamy's socioeconomic status scale}

The original version of Kuppuswamy's socioeconomic scale (SES) developed in 1976 in India (Kuppuswamy, 1981) was revised by Kumar et al in 2012. It is an important tool to measure SES in urban communities. (Kumar et al., 2012). It consists of three variables: family head's education, occupation and monthly family income. Based on the overall score, SES was categorized as follows: lower, lower middle and upper middle (Kumar et al., 2012).

\section{Family size and family type}

Family members were the persons living in the same household and shared the same kitchen. The family size was categorized into small ( $\leq 4$ members), medium (5-6 members) and large ( $\geq 7$ members) depending on the family members. The families were of two types: (i) nuclear family consisting of husband, wife and their children; and (ii) extended family - where more than one nuclear family live together and shares the household functions and income (Georgas et al., 2001).

\section{Maternal occupation}

Maternal occupation was categorized as housewives and working mothers. The women were considered working mothers if they worked outside the home for income in addition to the work they performed at home. The mothers were considered housewives if they were not involved in any income generating-activity outside of the home.

\section{Child feeding index}

Child feeding practice was measured using the age-specific child feeding index for 0-6, 6-9, 9-12, 12-36 and 36 60 month age groups. The variables used in the index creation were breastfeeding, use of baby feeding in the previous 24 hours, dietary diversity, food group frequency and meal frequency. The general scoring system was to assign a score of -1 for a potentially harmful practice, a score of 0 for medium practice and a score of 1 for a positive practice. The final child feeding index was the sum of the scores obtained for each variable (Arimond \& Ruel, 2001). Feeding terciles were derived to categorize feeding practices into poor, average, and good to make them comparable across the age groups. 


\section{Hygiene spot check observation}

Child's and mother's hygiene was assessed based on a hygiene spot check of the general appearance of hands, clothes, face and hair (Ruel \& Arimond, 2002). Each observation was scored 0 or 1 which indicates dirty and clean, respectively. The sum of scores generated an overall hygiene scale (range: 0-4) where higher scores indicated a higher level of cleanliness and vice versa (Nguyen et al., 2014). Hygiene spot check observation was performed from all the participants by a single team of data collectors to minimize subjectivity.

\section{Preventive health seeking index}

Preventive health care service use was assessed by the preventive health seeking index. This index included three variables, i.e., whether the child had been taken to growth monitoring in the previous month, and whether the child had received pentavalent and measles immunizations. A score of -1 was given for children who had not received the immunization or had not attended growth monitoring in the previous month, and 0 was given for those who had done so. The index scores ranged from -2 to 0 (Armar-Klemesu, Ruel, Maxwell, Levin, \& Morris, 2000). As a child becomes eligible for the specific vaccine when it reaches a certain age, the variables related to immunization (i.e., whether the child had received BCG, Pentavalent, OPV and measles) were included in the index for the relevant age groups.

\section{Child illness}

Child was considered ill if s/he had symptoms of either diarrhea or acute respiratory infection (ARI) within 30 days as per maternal recall.

\section{Maternal nutritional status}

According to BMI maternal nutritional status was measured. Undernutrition was considered if her BMI was $<18.5 \mathrm{~kg} / \mathrm{m}^{2}$ (Barba, Cavalli-Sforza, Cutter, \& Darnton-Hill, 2004).

\section{Child nutritional status}

Child nutritional status was assessed as per WHO recommended length/height-for age Z-score (HAZ), weightfor-height Z-score (WHZ) and weight-for-age Z-score (WAZ). The child was defined as stunted, wasted or underweight if his or her HAZ, WHZ or WAZ, respectively, was less than -2 standard deviations (SD) (De Onis, 2006).

\section{Conceptual framework}

The conceptual framework of this study is shown in Figure 1.

\section{Data collection technique}

Data were collected by face-to-face interviews, measurements of height and weight and observations of cleanliness of the child and mother. The interview was conducted at the participant's home ensuring the privacy and confidentiality. Informed written consent was obtained before the interview. Anthropometric measurements were performed following standard procedures (Cogill, 2003). Child weight was measured using electronic 
scales accurate to $100 \mathrm{~g}$. Supine length was taken up to the age of 24 months and standing height was taken after 24 months using locally manufactured length/height boards that were precise to $1 \mathrm{~mm}$. The height and weight of the mothers were also measured. Their hands, clothes, face and hair were observed for cleanliness. Data were collected from all the participants by a single team of data collectors.

\section{Data analysis}

Data analysis began with descriptive analysis. Means and SD were calculated for continuous variables, while frequencies and percentages were calculated for categorical variables. HAZ, WHZ and WAZ were calculated from the child's age, height and weight using the software 'WHO Anthro' (World Health Organization, 2011). Bivariate analysis was performed to determine the factors associated with undernutrition. The variables of interest in the bivariate analysis were the child's age and sex, maternal age, education occupation and nutritional status, SES, household food security, monthly family income, family type, family size, number of children under five years of age in the household and maternal mental health. Bivariate analysis was also performed to determine the association between maternal mental health and childcare practices and between childcare practices and child nutritional status. $\chi^{2}$-test, Fisher's exact test, Mann-Whitney U test and Spearman correlation were carried out as appropriate. To determine the association between maternal mental health and child nutritional status, a $\chi^{2}$ test was performed, and multivariate logistic regression was conducted after controlling for the other factors that showed significant association in bivariate analyses. Statistical significance was defined as $p<0.05$. Data analysis was performed using IBM SPSS version 21.0.

\section{Results}

\section{Sociodemographic characteristics}

The sociodemographic characteristics of the respondents are described in Table 1. Infants were less common (14.0\%) than other age groups whereas the age group of 24-35 months consisted of maximum number of children (31.4\%). Sex distribution was almost equal with a ratio of 1:1.2. The age of the mothers ranged from 15 to 44 years with a mean of $25.30 \pm 5.65$ years. Maximum mothers were educated up to primary level $(40.9 \%)$, were housewives (89.8\%) and belonged to low socioeconomic status (64.4). More than half $(52.7 \%)$ of the respondents were fallen at moderately food insecure state and only one-tenth (12.9\%) were at food secure state. The monthly family income ranged from 2,000 BDT to 50,000 BDT with an average of $10595 \pm 5234$ BDT. Most of the respondents belonged to a nuclear family (83\%). The total family member ranged from 3 to 12 and the family size of the maximum respondents was small (59.5\%).

\section{Prevalence of poor maternal mental health}

The prevalence of maternal CMD was $46.2 \%$. Household food insecurity and maternal undernutrition were the factors of maternal CMD. The detailed findings have been published elsewhere (Khan \& Flora, 2017). 


\section{Prevalence of child stunting, wasting and underweight}

Out of 264 children, $117(44.3 \%)$ were stunted, 48 (18.2\%) were wasted and $89(33.7 \%)$ were underweight.

\section{Maternal mental health, childcare practice and child illness}

The associations of maternal mental health with child feeding practice, hygiene practice, preventive care service use and child illness are shown in Table 2. Out of 264 children, good, average and poor feeding practice was $25.8 \%, 48.5 \%$ and $25.7 \%$, respectively. The proportion of poor feeding practices was more than three times higher among the mothers having CMD (41.8\%) than those having no CMD (12.0\%). The mean child's hygiene score was lower $(2.57 \pm 1.02)$ in mothers having CMD than in those having no CMD (3.42 \pm 0.85$)$. Similarly, the mother's hygiene score was lower in mothers with CMD (3.30 \pm 0.79$)$ than in those with no CMD (3.77 \pm $0.55)$. The mean preventive care score was less $(-1.24 \pm 0.53)$ among the mothers whose mental health was poor than whose mental health was normal $(-1.08 \pm 0.50)$. Diarrheal disease was more common among the children of the mothers with CMD (54.4\%) than normal mother (38.6\%).

\section{Childcare practice and nutritional status}

The associations of child feeding practices and child illness with child nutritional status are illustrated in Table 3. Child stunting was less with high feeding practice $(35.3 \%)$ and higher with low feeding practice $(54.4 \%)$. Proportion of wasting was two times higher among children with medium feeding practice (18.0\%) and four times higher among the children with low feeding practice $(29.2 \%)$ than the children with high feeding practice (7.4\%). Child underweight was about three times and five times more common among the children with medium $(35.9 \%)$ and low feeding practice $(52.9 \%)$, respectively, than the children with high feeding practice $(10.3 \%)$. Both HAZ and WAZ score increased with the child's hygiene score and mother's hygiene score increased. No relationship was noticed between preventive care service use and HAZ, WHZ and WAZ. Proportion of stunting, wasting or underweight was more common in the children who had a history of diarrhea within 30 days than who did not have.

\section{Sociodemographic characteristics and child nutritional status}

The associations of child characteristics, maternal characteristics and household characteristics with HAZ, WHZ and WAZ are shown in Table 4. Child age $(p=0.002)$ and sex $(p<0.001)$ was associated with child stunting. Stunting was less common among the male children $(26.2 \%)$ than female ones $(59.9 \%)$. The percentage of stunted children was highest (54.4\%) in the 12-23 months age group and lowest (12\%) among under-six months. The factors associated wasting were child sex $(p=0.001)$ and number of under-five children in the household $(p=0.001)$. The percentage of wasting was higher in female children $(25.4 \%)$ than in male children $(9.8 \%)$. Wasting was more common in the families which had more than one child (38.2\%) than in those having single child $(15.5 \%)$. The factors contributing to child underweight were child sex $(p<0.001)$, maternal nutrition ( $p=0.001)$ and household food insecurity $(p=0.001)$. The percentage of wasting was about three times higher in female children $(25.4 \%)$ than in male children $(9.8 \%)$. Child underweight was more common in case of 
underweight mothers $(52.6 \%)$ than the normal mothers $(28.5 \%)$. The proportion of the underweight children increased with the increased level of food insecurity. It was highest in severely food insecure households $(50.0 \%)$ and lowest in food secure households (14.7\%).

\section{Maternal mental health and child nutritional status}

\section{Maternal mental health and child stunting}

Child stunting was more common in the mothers with CMD (50.0\%) than in the mothers with no CMD (39.4\%). This difference was not statistically significant $(p=0.085$, Table 5)

\section{Maternal mental health and child wasting}

Approximately one-fourth of mothers (25.4\%) with CMD had wasted children, whereas only $12 \%$ of mothers with no CMD had wasted children which was statistically significant $(p=0.005)$. The logistic regression model contained three independent variables (maternal mental health, child sex and number of under-five children in the household). The final model was statistically significant $(p<0.001)$. Mothers with CMD were approximately two times more likely to have wasted children than mothers with no CMD after adjusting for the effect of child sex and number of children under five in the household (adjusted $\mathrm{OR}=2.25,95 \% \mathrm{CI}=1.15-4.43$, Table 5)

\section{Maternal mental health and child underweight}

The underweight children were more common in mothers with CMD (45.1\%) than in mothers with no CMD $(23.9 \%)$. This difference was statistically significant $(p<0.001)$. The logistic regression model contained four independent variables (maternal mental health, maternal nutritional status, child sex and household food security). The final model was statistically significant $(p<0.001)$. The association between maternal CMD and child underweight was attenuated and no longer statistically significant after controlling for the effect of maternal nutrition, child sex and household food security (adjusted OR=1.77, 95\% CI=0.94-3.33, Table 5).

\section{Discussion}

This study revealed maternal CMD as a considerable public health problem that was associated with child undernutrition. Approximately half of the mothers (46.2\%) were identified as suffering from CMD in this study. This result is similar (49\%) to a previous study carried out in Bangladesh (Nguyen et al., 2014). Although maternal CMD is very common in developing countries, its prevalence differs from country to country: Vietnam 31.2\%; Ethiopia 39.4\% (Nguyen et al., 2014); Peru 30\% and India 30.0\% (Harpham et al., 2005). The high prevalence of maternal CMD in developing countries might be due to lower socioeconomic conditions, food insecurity, being younger, being illiterate, undernutrition, unsupportive partners and experiencing physical violence (Fisher et al., 2012; Nguyen et al., 2014).

Childcare practices are identified as a key underlying cause of child malnutrition. The mental health of caregivers is one of the components that affects child care practice, as stated in the UNICEF care model (World Health Organization, 2004). Several studies have revealed that maternal mental health adversely affects child feeding practices resulting in malnutrition (Harpham et al., 2005; Nguyen et al., 2014). In the present study, 
childcare practices were measured in three domains - feeding practice, hygiene practice and preventive care service use. Maternal mental health was found to be associated with all three domains. Maternal CMD could prevent them from taking proper care of their children, especially infant feeding, improper food preparation and child care regarding immunization, and therefore, it constitutes a risk factor for impairment of the nutritional status of the children (Rahman et al., 2004; R. C. Stewart, 2007).

This study found an association of child feeding practices with wasting and underweight. A study conducted in Latin America also found an association between child feeding practices and nutritional status (Ruel \& Menon, 2002). The important factors of hygiene practice are social, lifestyle, and environmental factors, which are indirectly associated with the mental health of family members (Sherriff \& Golding, 2002). A significant relationship was observed between hygiene practice and child stunting and wasting. A similar finding was observed in our analysis of the relationship between individual hygiene and child nutrition (Ruel \& Menon, 2002). This might be because of inadequate hygiene practices increase the risk of infection among children especially diarrhea, helminth infection and other gastrointestinal symptoms (Aiello \& Larson, 2002). Harpham et al. have also described a pathway showing how maternal common mental disorder affects child nutrition through breast feeding, child immunization and child physical health (Harpham et al., 2005).

The association between maternal CMD and child illness was examined in this study. Diarrheal disease was significantly associated with maternal CMD. To our knowledge, few studies have examined the association between maternal mental health and child illness (Humphreys, Araya, Cruchet, Espinoza, \& Brunser, 1996; Nguyen et al., 2014; Rahman, Bunn, Lovel, \& Creed, 2007). The association between maternal CMD and diarrhea can be explained by poor maternal mental health affecting the ability of mothers to take on their personal hygiene which translates into diarrheal diseases among children. These findings suggest that a potential pathway for the relationship between poor maternal mental health and child undernutrition could be through child illness. Poor mental health may hinder the mother's ability to take adequate care of her child, prevent illness and seek health care when the child is ill.

Out of 264 children, $44.3 \%$ were stunted, $18.2 \%$ were wasted and $33.7 \%$ were underweight. These findings are consistent with the national data where the prevalence of stunting, wasting and underweight are $41 \%, 16 \%$ and $36 \%$, respectively (NIPORT et al., 2012). Evidence of an association between maternal CMD and poor nutritional status in the urban population of Bangladesh in this study confirmed previous evidence from other countries (Harpham et al., 2005; Medhin et al., 2010; Nguyen et al., 2014; Robert C Stewart et al., 2008). After controlling for the effects of the confounders, a significant association was found between maternal CMD and child wasting. An association was found with underweight in the bivariate analysis, but it was attenuated and no longer statistically significant in the multivariate analysis suggesting that other factors might be more important or so tightly correlated with maternal mental health that the independent effects cannot be disentangled in this model. Surprisingly, there was no statistically significant association between maternal CMD and stunting, which is hard to explain. Stunting is a condition that reflects the cumulative effects of chronic malnutrition, while wasting is a condition that reflects acute or recent nutritional deficits. Underweight reflects the combination of acute and chronic malnutrition. The SRQ-20, used to assess maternal mental health is based on some symptoms in the previous 30 days. Therefore, the association of maternal CMD with wasting, which was found in this study, is justifiable. However, this result is inconsistent with the results of Harpham et 
al. and Nguyen et al. Maternal CMD is associated with child stunting in India and with child underweight in Vietnam (Harpham et al., 2005). A study conducted in Bangladesh found that maternal CMD was associated with stunting but no association with wasting or underweight (Nguyen et al., 2014). The variability of findings may be explained by sociocultural differences in care and feeding practices, differences in maternal education, socioeconomic status, household food security and other factors. This heterogeneity in the results is particularly interesting and needs to be further analyzed to fully understand this relationship.

Despite all efforts, there were some limitations in this study. First, the study was conducted in a selected urban area. Therefore, the study result might not necessarily have external validity. Second, no psychometric validation was performed on the translated version of the questionnaires used for this study. Also, the SRQ-20 is a screening tool to assess mental health, not a diagnostic tool. Therefore, the number of mothers identified as having CMD might differ from the actual number. Third, data were collected based on maternal recall of their mental distress in the past four weeks prior to the interview. Maternal recall can result in either under- or over-reporting. Fourth, the association of childcare practice with maternal mental health and child nutritional status was investigated by bivariate analysis. The logistic regression model was not used to address this. Last, as it was a cross sectional study, a causal relationship between maternal mental health and child nutrition was not established. The potential reverse causality cannot be ruled out as poor child health is a contributor to maternal CMD, and this may be especially true when children are acutely undernourished rather than chronically moderately undernourished. This in turn may impede the ability of the mother to take adequate care of the child.

The study results may contribute to understanding the importance of maternal mental health as a public health problem and its potential pathways to cause child malnutrition. It is the time to invest to fully understand risk factors for CMD and develop evidence-based mental health interventions to improve maternal mental health. Maternal mental health components need to be incorporated into child nutrition programs to improve child nutritional status.

\section{References}

Aiello, A. E., \& Larson, E. L. (2002). What is the evidence for a causal link between hygiene and infections? Lancet Infect Dis, 2(2), 103-110.

Arimond, M., \& Ruel, M. T. (2001). Assessing care: progress towards the measurement of selected childcare and feeding practices, and implications for programs. Food Consumption and Nutrition Division Discussion Paper, 119.

Armar-Klemesu, M., Ruel, M. T., Maxwell, D. G., Levin, C. E., \& Morris, S. S. (2000). Poor maternal schooling is the main constraint to good child care practices in Accra. The Journal of nutrition, 130(6), 1597-1607.

Ashaba, S., Rukundo, G. Z., Beinempaka, F., Ntaro, M., \& LeBlanc, J. C. (2015). Maternal depression and malnutrition in children in southwest Uganda: a case control study. BMC public health, 15, 1303. doi:10.1186/s12889-015-2644-y 
Barba, C., Cavalli-Sforza, T., Cutter, J., \& Darnton-Hill, I. (2004). Appropriate body-mass index for Asian populations and its implications for policy and intervention strategies. The Lancet, 363(9403), 157.

Barsisa, B., Derajew, H., Haile, K., Mesafint, G., \& Shumet, S. (2021). Prevalence of common mental disorder and associated factors among mothers of under five year children at Arbaminch Town, South Ethiopia, 2019. PloS one, 16(9), e0257973-e0257973. doi:10.1371/journal.pone.0257973

Black, R. E., Victora, C. G., Walker, S. P., Bhutta, Z. A., Christian, P., De Onis, M., . . Martorell, R. (2013). Maternal and child undernutrition and overweight in low-income and middle-income countries. The Lancet, 382(9890), 427-451.

Brown, K. H. (2003). Diarrhea and malnutrition. The Journal of nutrition, 133(1), 328S-332S.

Carvalhaes, M. A., \& Benicio, M. H. (2006). Malnutrition in the second year of life and psychosocial care: a case-control study in an urban area of Southeast Brazil. Cad Saude Publica, 22(11), 2311-2318.

Cavalcante-Neto, J. L., Paula, C. S., Florencio, T. M., \& Miranda, C. T. (2016). Disability due to maternal common mental disorders (CMDs) as a risk factor for chronic childhood malnutrition: cross-sectional study. Sao Paulo Med J, 134(3), 228-233. doi:10.1590/15163180.2015 .02342112

Coates, J., Swindale, A., \& Bilinsky, P. (2007). Household Food Insecurity Access Scale (HFIAS) for measurement of food access: indicator guide. Washington, DC: Food and Nutrition Technical Assistance Project, Academy for Educational Development.

Cogill, B. (2003). Anthropometric indicators measurement guide. Food and Nutrition Technical Assistance Project. Retrieved from Washington, DC:

De Onis, M. (2006). WHO Child Growth Standards: Length/height-for-age, weight-for-age, weightfor-length, weight-for-height and body mass index-for-age: Methods and development.

Fisher, J., Cabral de Mello, M., Patel, V., Rahman, A., Tran, T., Holton, S., \& Holmes, W. (2012). Prevalence and determinants of common perinatal mental disorders in women in low- and lower-middle-income countries: a systematic review. Bull World Health Organ, 90(2), 139g149g. doi:10.2471/blt.11.091850

Georgas, J., Mylonas, K., Bafiti, T., Poortinga, Y. H., Christakopoulou, S., Kagitcibasi, C., . . Orung, S. (2001). Functional relationships in the nuclear and extended family: A 16-culture study. International Journal of Psychology, 36(5), 289-300. 
Harpham, T., Huttly, S., De Silva, M. J., \& Abramsky, T. (2005). Maternal mental health and child nutritional status in four developing countries. Journal of epidemiology and community health, 59(12), 1060-1064.

Humphreys, D., Araya, M., Cruchet, S., Espinoza, J., \& Brunser, O. (1996). Maternal neurotic symptoms and infants' risk of developing persistent diarrhoea. Revista de Saúde Pública, 30(3), 213-217.

Khan, A. M., \& Flora, M. S. (2017). Maternal common mental disorders and associated factors: a cross-sectional study in an urban slum area of Dhaka, Bangladesh. International Journal of Mental Health Systems, 11(1), 23.

Kumar, N., Gupta, N., \& Kishore, J. (2012). Kuppuswamy's socioeconomic scale: updating income ranges for the year 2012. Indian journal of public health, 56(1), 103-104.

Kuppuswamy, B. (1981). Manual of socioeconomic status (urban). Delhi: Manasayan, 8.

Medhin, G., Hanlon, C., Dewey, M., Alem, A., Tesfaye, F., Lakew, Z., . . Tomlinson, M. (2010). The effect of maternal common mental disorders on infant undernutrition in Butajira, Ethiopia: the P-MaMiE study. BMC psychiatry, 10(1), 32.

Nguyen, P. H., Saha, K. K., Ali, D., Menon, P., Manohar, S., Mai, L. T., ... Ruel, M. T. (2014). Maternal mental health is associated with child undernutrition and illness in Bangladesh, Vietnam and Ethiopia. Public Health Nutrition, 17(6), 1318-1327.

NIPORT, Mitra and Associates, \& ICF International. (2012). Bangladesh Demographic and Health Survey 2011. Retrieved from

Olson, C. M., Strawderman, M. S., \& Dennison, B. A. (2009). Maternal weight gain during pregnancy and child weight at age 3 years. Maternal and child health journal, 13(6), 839.

Parcells, D. (2010). Women's mental health nursing: depression, anxiety and stress during pregnancy. Journal of psychiatric and mental health nursing, 17(9), 813-820.

Pongou, R., Ezzati, M., \& Salomon, J. A. (2006). Household and community socioeconomic and environmental determinants of child nutritional status in Cameroon. BMC public health, 6(1), 98.

Prince, M., Patel, V., Saxena, S., Maj, M., Maselko, J., Phillips, M. R., \& Rahman, A. (2007). No health without mental health. Lancet, 370(9590), 859-877. doi:10.1016/s0140-6736(07)61238-0

Rahman, A., Bunn, J., Lovel, H., \& Creed, F. (2007). Maternal depression increases infant risk of diarrhoeal illness:-a cohort study. Archives of Disease in Childhood, 92(1), 24-28.

Rahman, A., Iqbal, Z., Bunn, J., Lovel, H., \& Harrington, R. (2004). Impact of maternal depression on infant nutritional status and illness: a cohort study. Archives of general psychiatry, 61(9), 946. 
Rayhan, M. I., \& Khan, M. S. H. (2006). Factors causing malnutrition among under five children in Bangladesh. Pak J Nutr, 5(6), 558-562.

Ruel, M. T., \& Arimond, M. (2002). Spot-check observational method for assessing hygiene practices: review of experience and implications for programmes.

Ruel, M. T., \& Menon, P. (2002). Child feeding practices are associated with child nutritional status in Latin America: innovative uses of the demographic and health surveys. The Journal of nutrition, 132(6), 1180-1187.

Santos, D. S., Santos, D. N., Silva Rde, C., Hasselmann, M. H., \& Barreto, M. L. (2011). Maternal common mental disorders and malnutrition in children: a case-control study. Soc Psychiatry Psychiatr Epidemiol, 46(7), 543-548. doi:10.1007/s00127-010-0220-4

Sherriff, A., \& Golding, J. (2002). Factors associated with different hygiene practices in the homes of 15 month old infants. Arch Dis Child, 87(1), 30-35.

Smith, L. C., \& Haddad, L. J. (2000). Explaining child malnutrition in developing countries: A crosscountry analysis (Vol. 111): Intl Food Policy Res Inst.

Steel, Z., Marnane, C., Iranpour, C., Chey, T., Jackson, J. W., Patel, V., \& Silove, D. (2014). The global prevalence of common mental disorders: a systematic review and meta-analysis 1980-2013. Int J Epidemiol, 43(2), 476-493. doi:10.1093/ije/dyu038

Stewart, R. C. (2007). Maternal depression and infant growth: a review of recent evidence. Matern Child Nutr, 3(2), 94-107. doi:10.1111/j.1740-8709.2007.00088.x

Stewart, R. C., Umar, E., Kauye, F., Bunn, J., Vokhiwa, M., Fitzgerald, M., . . Creed, F. (2008). Maternal common mental disorder and infant growth-a cross-sectional study from Malawi. Maternal \& child nutrition, 4(3), 209-219.

Surkan, P. J., Kennedy, C. E., Hurley, K. M., \& Black, M. M. (2011). Maternal depression and early childhood growth in developing countries: systematic review and meta-analysis. Bull World Health Organ, 89(8), 608-615. doi:10.2471/blt.11.088187

UNICEF. (2009). Child and Maternal Nutrition in Bangladesh. Retrieved from http://www.unicef.org/bangladesh/Child and Maternal Nutrition(1).pdf

World Health Organization. (1994). A user's guide to the Self Reporting Questionnaire (SRQ). Geneva: World Health Organization.

World Health Organization. (2004). The importance of caregiver-child interactions for the survival and healthy development of young children: A review. Retrieved from

World Health Organization. (2011). WHO Anthro (version 3.2.2, January 2011) and macros. Retrieved from http //www.who.int/child growth/software/en/ 
World Health Organization. (2012). Global Database on Child Growth and Malnutrition. Retrieved from http://www.who.int/nutgrowthdb/estimates2012/en/index.html 


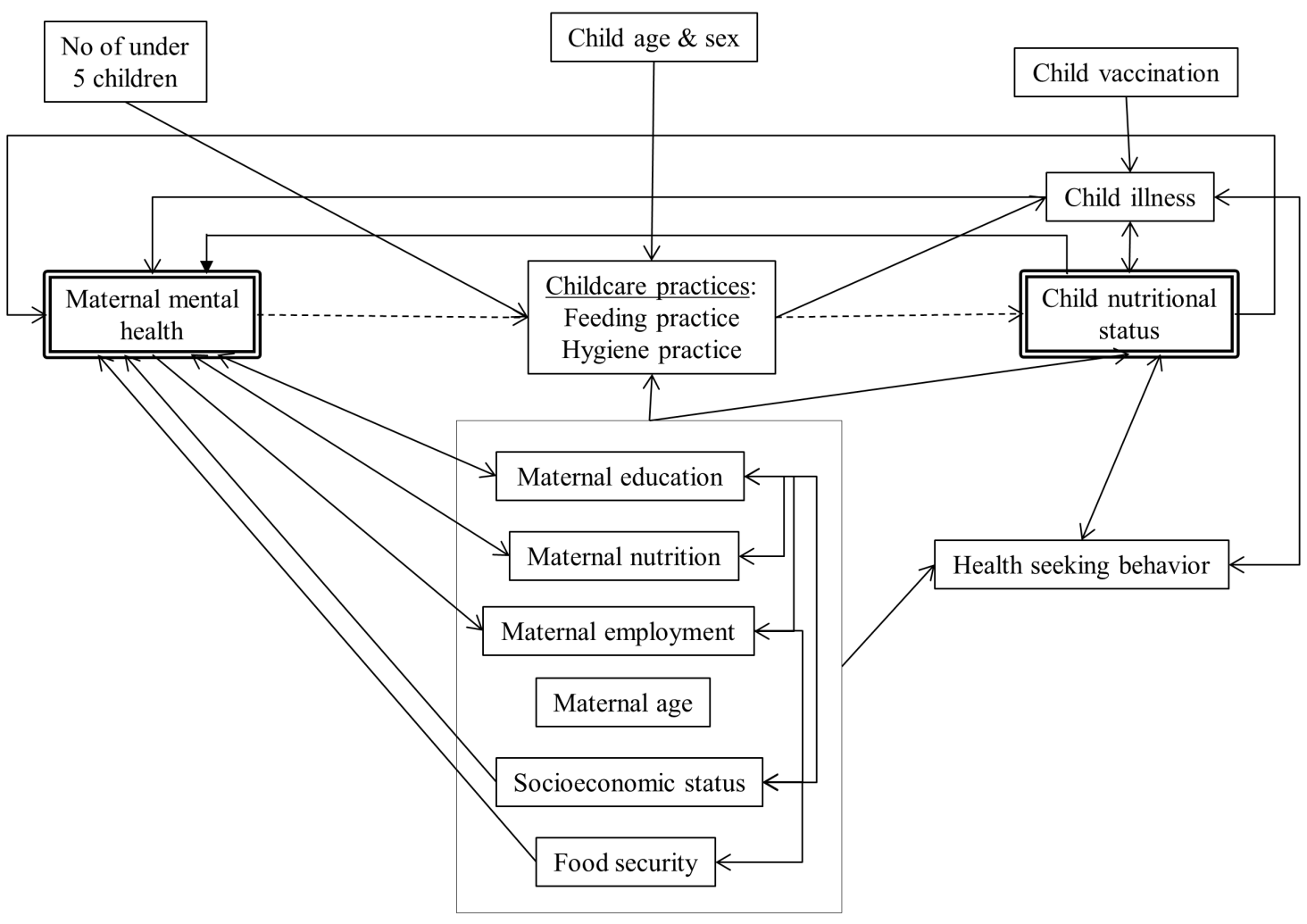

\section{Figure 1}

